\title{
Observed and Self-reported Hand Hygiene Compliances and Associated Factors among Healthcare Workers at a County Referral Hospital in Kenya
}

Karlmax Kiprotich ( $\square$ Karlmaxk@yahoo.com )

Moi University School of Public Health https://orcid.org/0000-0001-5293-5199

Atipatsa Chiwanda Kaminga

Central South University

Miriam Kessi

Central South University

Wang Honghong

Central South University

Research

Keywords: Hand hygiene, Compliance, Healthcare workers, Direct observation, Healthcare-associated infections

Posted Date: September 24th, 2020

DOI: https://doi.org/10.21203/rs.3.rs-79985/v1

License: (c) (1) This work is licensed under a Creative Commons Attribution 4.0 International License.

Read Full License

Version of Record: A version of this preprint was published at Scientific African on October 1st, 2021. See the published version at https://doi.org/10.1016/j.sciaf.2021.e00984. 


\section{Abstract}

Background: Hand hygiene is effective in preventing and controlling

healthcare-associated infections (HCAls); however, its compliance remains low,

and reasons are poorly understood, thus its research and feedback may lead to

successful interventions. This study aimed to explore the observed and self-reported hand hygiene compliance and associated factors among healthcare workers (HCWs) in the medical/surgical ward of a county referral hospital.

Methods: Cross-sectional study design was used in this study. Direct observation guided by 5 moments for hand hygiene concept was carried to describe compliance, and the questionnaire technique informed by Theoretical Domains Framework (TDF) was employed to investigate self-reported compliance, barriers, and facilitators of hand hygiene compliance. This study was done at a county referral hospital in Kenya. The total number of hand hygiene opportunities observed was 347 among $55 \mathrm{HCWs}$, and $132 \mathrm{HCWs}$ filled the questionnaires.

Results: The overall compliance based on direct observation was $23.9 \%$, whereas self-reported compliance was $80.0 \%$. Hand hygiene was significantly associated with indication $(p<0.001)$, training ( $p$ $=0.014)$, social influences $(p=0.002)$ and knowledge $(p=0.044)$. Barriers identified were environmental resources, social influences, beliefs about consequences, memory, attention, and decision processes. Facilitators included knowledge and social/professional role, and identity.

Conclusions: Despite the high report rate of hand hygiene compliance, the observed compliance is unacceptably low. Although knowledge, and social/professional role and identity of the HCWs may primarily facilitate hand hygiene compliance, barriers associated with non-compliance such as environmental resources remain significant constraints and should be considered to ensure optimal hand hygiene compliance and quality of patient care. The results also suggest that HCWs education on hand hygiene still needs improvements, particularly on the 5 moments for hand hygiene.

\section{Background}

Hand hygiene is an effective element of standard precautions in preventing and controlling the spread of healthcare-associated infections (HCAls) (1) and remains a priority indicator of the quality of healthcare (2). A variety of microorganisms that cause HCAl, such as methicillin-resistant Staphylococcus aureus, Escherichia coli, and Clostridium difficile (3) are transmitted by healthcare workers (HCWs) through their hands $(4,5)$. Evidence suggests that by performing hand hygiene, we break the chain of transmission of the pathogens between patients and the healthcare environment (6) and can result in a reduction of $15 \%$ to $30 \%$ of HCAls (7). Even though, hand hygiene compliance in low-income countries is as low as $21.1 \%$ due to the varied factors (8). 
Previous studies have informed that self-assessment using questionnaires often indicate exaggerated findings on hand hygiene compliance as compared to direct observation $(8,9)$. Therefore, WHO $(2009)$ has recommended direct observation based on the 5 moments for hand hygiene as a gold standard technique for monitoring hand hygiene practices among HCWs during patient care and remains widely used and reliable method (10). The 5 moments for hand hygiene states that there are five moments when HCWs should practice hand hygiene during patient care to prevent HCAls: 1) before touching a patient, 2) before a clean/aseptic procedure, 3 ) after exposure to body fluids risk, 4) after touching a patient, and 5) after touching patient surroundings (11).

Moreover, it has been pointed out that a behavior change theory-informed framework such as the theoretical domain framework (TDF) can be useful in assessing barriers and facilitators to hand hygiene compliance among HCWs (12). Whereas insufficient use of a behavioral theory to understand factors associated with hand hygiene compliance may lead to a suboptimal design of interventions (13).

In Kenya, there were inadequate observational studies on hand hygiene, and the compliance greatly varied (2.3-28\%) among HCWs (14-16). Most hospitals in resource-limited settings have combined medical and surgical wards. Still, there have been limited attempts to evaluate hand hygiene behavior in this department. Therefore, this study aimed to describe the current situation of hand hygiene compliance among HCWs at a medical/surgical ward based on both direct observation and questionnaire, and investigate demographic and behavioral factors associated with the hand hygiene compliance.

\section{Methods}

\section{Setting and participants}

This study was done at Kapsabet county referral hospital, Kenya. The hospital receives approximately 400 patients daily and has a bed capacity of 200. Between July and September 2019, data were collected from the medical/surgical ward due to its busyness and cruciality of hand hygiene. Each of the six rooms in the department had a sink with running water, and no soap dispensers and hand towels were available during the period of this study. Alcohol-based handrubs were also not accessible at the point of care, but some HCWs had personal pocket handrubs. The target group was all HCWs who provided patients' care, including nurses, patient care assistants (PCA), doctors, and other personnel (laboratory technicians, pharmacists, physiotherapists, and students).

\section{Sample size}

A sample of 200 opportunities for each healthcare unit per observation period is required to meet statistical significance and is the minimum number recommended by the WHO (17). A total of $55 \mathrm{HCWs}$ who qualified according to the inclusion criteria were engaged and all agreed to participate. Each of them was observed for four to eight hand hygiene opportunities during patient care and the final sample size was 347 opportunities. Out of $190 \mathrm{HCWs}$ in the ward, 143 who met the established inclusion criteria were 
invited. However, 11 declined to participate, citing being committed to work. Hence the final sample size for the valid questionnaire was 132 , and the response rate $92.3 \%$.

\section{Study design}

This was a quantitative cross-sectional study consisting of two techniques for data collection including direct observation of hand hygiene compliance during patient care, followed by a questionnaire survey of compliance, barriers, and facilitators.

\section{Direct observation}

A certified observer was further trained and tested in assessing compliance according to the observation guidelines before the actual observation through the WHO training film (18). The training was necessary to minimize observer bias. Selection bias was reduced by stratification of the participants and by random allocation of times of the day to make observations. Evidence suggests that the Hawthorne effect during hand hygiene observation is more evident in healthcare settings with high compliance and minor in low compliance settings. The reason being when HCW does not know when to perform hand hygiene, even in the presence of an observer, its effect on compliance is minor (19). A minimal Hawthorne effect was therefore expected due to low compliance in the setting. Chen et al. (2015) maintain that the Hawthorne effect may increase as soon as the observer starts observation but decreases as time contact between observer and HCWs increases as the HCWs become comfortable with the observer (20).

Data were collected data using a validated WHO observation form (2). All the HCWs in the medical/surgical ward were informed about the study and invited to participate one week before observation. However, the timing of when they would be observed was not disclosed. Overt observations were used in this study for the reason that it would have been challenging to accomplish covert observation in such a small setting. Hand hygiene compliance was assessed on the selected HCWs at the point of patient care. After filling the form header, each observed opportunity and the related hand hygiene actions were recorded.

During the observation session, the observer did not interfere with the observed HCW. One observation session ended after around 20 minutes. More than one session was required to achieve the targeted number of opportunities. These additional sessions were undertaken at an alternative time and date. If the observer was unsure whether the observed HCW did hand hygiene, then such a moment was not be recorded. Glove usage was only recorded when an opportunity occurs, and an HCW under observation missed hand hygiene. The observation was only performed during the day for logical reasons.

\section{Questionnaire}

A questionnaire informed by TDF was used in this study. The central concept of the questionnaire aimed to evaluate HCW's knowledge, social/professional role and identity, beliefs about hand hygiene consequences, hand hygiene resources, social influences and memory, attention, and decision processes as the potential behavior change domains that determine compliance. Responses were evaluated using a 
5-point Likert scale indicating strongly disagree, disagree, neither disagree/agree, agree, and strongly agree. The questionnaire was piloted with $15 \mathrm{HCWs}$ working in the hospital to assess comprehensibility, practicability, and acceptability. Proper adjustments were made, including simplification of language and elimination of structured questions as recommended by the piloted HCWs.

HCWs from different professional categories in the medical/surgical ward were identified and informed about the purpose and the expected duration of the survey (less than 15 minutes). Upon acceptance and giving consent, a questionnaire was issued for filling. A 1.43 US Dollar gift was given to each respondent in appreciation of their time. The reliability of the survey was determined by Cronbach's alpha to be 0.7 .

\section{Data analysis}

Data were analyzed using SPSS (IBM Corp. Released 2015. IBM SPSS Statistics for Windows, Version 23.0. Armonk, NY: IBM Corp.) at a $95 \%$ significance level. Observed compliance was determined as the percentage of the number of times hand hygiene was performed over the total number of opportunities. Chi-square Pearson's test was used to investigate the difference in the observed compliance rate across indications and demographics.

Questionnaire data were tested for normality before further analysis by the Shapiro-Wilk test which showed that the rates of hand hygiene compliance were not normally distributed $(p<0.001)$. In the same way as previous studies, the cut-off points of $60 \%$ and $80 \%$ grouped the cumulative scores of the six behavior change domains into low $(<60 \%)$, moderate $(60-80 \%)$ and high $(\geq 80 \%)(21,22)$. The low and moderate levels identified barriers while high levels of the domains defined facilitators. The KruskalWallis test was used to compare the mean score of self-reported compliance among the categories of HCWs' demographics and the domains.

\section{Results}

\section{General Information of the Participants}

The majority of HCWs observed were females (60.0\%), nurses (45.5\%), having a diploma (40.0\%), and had worked for below 5 years (41.8\%). Furthermore, over $50 \%$ (132/190) of HCWs providing direct healthcare in the department answered the questionnaire where the majority were also females (61.4\%) and had a diploma (56.1\%). Those who had worked for five years and below were also the majority in the questionnaire group (65.2\%) and had attended hand hygiene training at most once a year (65.4\%) Table 1.

Table 1. Descriptive statistics for demographic variables of the participants 


\begin{tabular}{|c|c|c|c|}
\hline \multirow[t]{2}{*}{ Item } & \multirow[t]{2}{*}{ Characteristics } & \multirow{2}{*}{$\begin{array}{l}\text { Observation }(\mathrm{N}=55) \\
\text { Frequency } \mathrm{n}(\%)\end{array}$} & \multirow{2}{*}{$\begin{array}{l}\text { Questionnaire }(\mathrm{N}=132) \\
\text { Frequency } \mathrm{n}(\%)\end{array}$} \\
\hline & & & \\
\hline \multirow[t]{4}{*}{ Age } & $<25$ years & $14(25.5)$ & $40(30.3)$ \\
\hline & 26-35 years & $17(30.9)$ & $41(31.1)$ \\
\hline & $36-45$ years & $12(21.8)$ & $23(17.4)$ \\
\hline & $>45$ years & $12(21.8)$ & $28(21.2)$ \\
\hline \multirow[t]{2}{*}{ Gender } & Male & $22(40.0)$ & $51(38.6)$ \\
\hline & Female & $33(60.0)$ & $81(61.4)$ \\
\hline \multirow[t]{4}{*}{ Education } & Certificate & $10(18.2)$ & $12(9.1)$ \\
\hline & Diploma & $22(40.0)$ & $74(56.1)$ \\
\hline & Degree & $15(27.3)$ & 38 (28.8) \\
\hline & Masters & 8 (14.5) & $8(6.1)$ \\
\hline \multirow[t]{4}{*}{ Profession } & PCA & $10(18.2)$ & $10(7.6)$ \\
\hline & Nurse & $25(45.5)$ & $37(28.0)$ \\
\hline & Physician & $14(25.5)$ & $36(27.3)$ \\
\hline & Others & $6(10.9)$ & $49(37.1)$ \\
\hline \multirow[t]{3}{*}{ Working years } & $<5$ years & $23(41.8)$ & $86(65.2)$ \\
\hline & $6-10$ years & $20(36.4)$ & $24(18.2)$ \\
\hline & $>10$ years & $12(21.8)$ & $22(16.7)$ \\
\hline \multirow[t]{3}{*}{ Training/yr. } & 0-1 Times/years & - & $85(65.4)$ \\
\hline & 2-3 Times/years & & 22 (16.9) \\
\hline & $>4$ Times/years & & $23(17.7)$ \\
\hline
\end{tabular}

Abbreviation: PCA, patient care assistants

\section{Hand hygiene compliance by direct observation}

Three hundred and forty-seven opportunities were observed during patient care and the overall compliance was $23.9 \%$. Handwashing was more common (73.5\%) than handrub (26.5\%) and HCWs, following non-compliance, were more likely to use gloves (52.7\%) than not. The compliance exceeded $30 \%$ after body fluid exposure and after touching a patient and was the lowest after touching patient surroundings (6.0\%). Pearson-Chi square test indicated that compliance was significantly higher after than before patient contact and lowest after touching patient environment $(p<0.001)$. There was no 
significant difference in compliance across demographics of HCWs such as working years, age, gender, education, and profession ( $p>0.05$ ) (Table 2).

Table 2. Observed compliance by indication and demographics

\begin{tabular}{|c|c|c|c|c|}
\hline Factors & $\begin{array}{l}\text { Total opportunities } \\
(\mathrm{N}=347)\end{array}$ & $\begin{array}{l}\text { HH performed } \\
(\mathrm{n}=83)\end{array}$ & $\begin{array}{l}\text { Compliance (\%) } \\
(23.92)\end{array}$ & P-value \\
\hline Indication & & & & $<0.001$ \\
\hline Before touching a patient & 62 & 11 & 17.7 & \\
\hline Before aseptic procedure & 80 & 16 & 20.0 & \\
\hline After body fluid exposure & 71 & 23 & 32.4 & \\
\hline After touching a patient & 67 & 29 & 43.3 & \\
\hline After touching surrounding & 67 & 4 & 6.0 & \\
\hline \multicolumn{5}{|l|}{ Demographic } \\
\hline Working years: & & & & 0.525 \\
\hline$<5$ & 154 & 38 & 24.7 & \\
\hline $6-10$ & 115 & 30 & 26.1 & \\
\hline$>11$ & 78 & 15 & 19.2 & \\
\hline Age (years): & & & & 0.774 \\
\hline$<25$ & 82 & 20 & 24.4 & \\
\hline $26-35$ & 104 & 25 & 24.0 & \\
\hline $36-45$ & 77 & 21 & 27.3 & \\
\hline$>46$ & 84 & 17 & 20.2 & \\
\hline Gender: & & & & 0.668 \\
\hline Male & 145 & 33 & 22.8 & \\
\hline Female & 202 & 50 & 24.8 & \\
\hline Education: & & & & 0.646 \\
\hline Certificate & 62 & 16 & 25.8 & \\
\hline Diploma & 141 & 30 & 21.3 & \\
\hline Degree & 97 & 27 & 27.8 & \\
\hline Masters & 47 & 10 & 21.3 & \\
\hline Profession: & & & & 0.566 \\
\hline PCA & 58 & 13 & 22.4 & \\
\hline Nurses & 159 & 41 & 25.8 & \\
\hline Physicians & 91 & 23 & 25.3 & \\
\hline Others & 39 & 6 & 15.4 & \\
\hline
\end{tabular}

Abbreviation: HH hand hygiene, PCA patient care assistants, $P=$ Chi-Square 
When HCWs were asked how often they thought they perform hand hygiene when required during patient care, they reported median compliance of $80.0 \%$. Kruskal-Wallis test indicated HCWs who attended hand hygiene training 2-3 times a year, had statistically significantly higher compliance than those who attended 0-1 time $(p=0.047)$. The compliance was however not significantly different across categories of age $(p=0.846)$, gender $(p=0.235)$, education $(p=0.539)$, profession $(p=0.769)$, and working years ( $=0.571)$. Data indicated that $85.3 \%$ of the HCWs who had an advanced level of hand hygiene knowledge reported significantly better compliance $(78.72 \%)$ as compared to those with moderate knowledge ( $p=$ 0.038). Similarly, more than $75 \%$ of the HCWs believed to have a high social/professional role and identity which facilitated compliance; however, compliance was not significantly associated with this domain $(p=0.925)$. On the other hand, the majority believed they had low $(54.6 \%)$ and moderate $(43.1 \%)$ social influences, while $2.3 \%$ reported high social influences, therefore this domain was considered a barrier to hand hygiene compliance. Those who had a higher level of social influence had significantly higher compliance ( $p=0.002) .2 .3 \%$ of the HCWs felt they had a high level of memory, attention, and decision processes, considering this factor to hinder compliance to hand hygiene. However, there was no significant relationship between compliance and memory, attention, and decision processes $(p=0.725)$. A small proportion of the HCWs (2.3\%) reported a high level of environmental context, resources, and time, this domain was therefore considered a barrier to compliance but the Kruskal-Wallis test indicated no significant association between compliance and this factor $(p=0.160)$. The beliefs about the consequences of performing hand hygiene were considered by the majority $(62.6 \%)$ to be intermediate, this factor was therefore classified as a barrier to compliance. There was no significant difference in compliance across the levels of beliefs about consequences $(p=0.156)$ (Table 3$)$.

Table 3. Self-reported hand hygiene compliance by demographics and behavior change domains 


\begin{tabular}{|c|c|c|c|c|c|}
\hline & Characteristic & $\begin{array}{l}(\mathrm{N}=132) \\
\text { Frequency } \mathrm{n} \\
(\%)\end{array}$ & $\begin{array}{l}\text { Mean HH } \\
\text { Compliance } \\
\%\end{array}$ & $\begin{array}{l}\mathrm{P}- \\
\text { value }\end{array}$ & Post-Hoc Test \\
\hline \multicolumn{6}{|l|}{ Demographic } \\
\hline \multirow[t]{4}{*}{ Age } & $<25$ & $40(30.3)$ & 77.2 & 0.846 & NA \\
\hline & $26-35$ & $41(31.1)$ & 77.9 & & \\
\hline & $36-45$ & $23(17.4)$ & 80.1 & & \\
\hline & $>46$ & $28(21.2)$ & 74.3 & & \\
\hline \multirow[t]{2}{*}{ Gender } & Male & $51(38.6)$ & 75.0 & 0.235 & NA \\
\hline & Female & $81(61.4)$ & 78.7 & & \\
\hline \multirow[t]{4}{*}{ Education } & Certificate & $12(9.1)$ & 74.6 & 0.539 & NA \\
\hline & Diploma & $74(56.1)$ & 78.9 & & \\
\hline & Degree & $38(28.8)$ & 76.5 & & \\
\hline & Masters & $8(6.1)$ & 70.6 & & \\
\hline \multirow[t]{4}{*}{ Profession } & PCA & $10(7.6)$ & 81.0 & 0.769 & NA \\
\hline & Nurse & $37(28.0)$ & 75.5 & & \\
\hline & Physician & 36 (27.3) & 78.4 & & \\
\hline & Others & 49 (37.1) & 77.1 & & \\
\hline \multirow[t]{3}{*}{ Working years } & $<5$ years & $86(65.2)$ & 77.6 & 0.571 & NA \\
\hline & $6-10$ years & $24(18.2)$ & 79.5 & & \\
\hline & $>11$ years & 22 (16.7) & 73.7 & & \\
\hline \multirow[t]{3}{*}{ Training/year } & 0-1 Times/yr. & $85(64.4)$ & 74.4 & 0.014 & $0-1$ vs $2-3$ \\
\hline & 2-3 Times/yr. & 22 (16.7) & 83.2 & & $p=0.047$ \\
\hline & $>4$ Times/yr. & $23(17.4)$ & 83.0 & & \\
\hline \multicolumn{6}{|l|}{ Behaviour Domain } \\
\hline \multirow[t]{4}{*}{ Social Influence } & Low & $71(54.6)$ & 72.83 & 0.002 & Low vs Moderate, $\mathrm{p}=$ \\
\hline & & & & & 0.005 \\
\hline & Moderate & $56(43.1)$ & 81.82 & & \\
\hline & High & $3(2.3)$ & 89.67 & & \\
\hline Memory, attention, & Low & $63(48.8)$ & 78.73 & 0.725 & NA \\
\hline \multirow{3}{*}{$\begin{array}{l}\text { \& decision } \\
\text { processes }\end{array}$} & Moderate & $63(48.8)$ & 76.63 & & \\
\hline & & & & & \\
\hline & High & $3(2.3)$ & 79.67 & & \\
\hline Environmental & Low & $73(56.2)$ & 76.14 & 0.160 & NA \\
\hline \multirow[t]{2}{*}{ resources \& time } & Moderate & $54(41.5)$ & 78.04 & & \\
\hline & High & $3(2.3)$ & 91.67 & & \\
\hline \multirow[t]{3}{*}{ Knowledge } & Low & $2(1.6)$ & 75.00 & 0.044 & Moderate vs High, $\mathrm{p}=$ \\
\hline & & & & & 0.038 \\
\hline & Moderate & $17(13.2)$ & 64.88 & & \\
\hline
\end{tabular}




\begin{tabular}{llllll} 
& High & $110(85.3)$ & 78.72 & & \\
\hline Social/professional & Low & $2(1.5)$ & 75.00 & 0.925 & NA \\
\hline role and identity & Moderate & $30(23.1)$ & 74.27 & & \\
\hline Beliefs about & High & $98(75.4)$ & 77.90 & & \\
consequences & Low & $19(15.4)$ & 69.21 & 0.156 & NA \\
& Moderate & $77(62.6)$ & 77.09 & & \\
\hline
\end{tabular}

Abbreviation: $H H$ hand hygiene, $P=$ Kruskal-Wallis, NA not applicable

\section{Discussion}

Our results indicated that self-reported compliance (80.0\%) was much higher compared to compliance by observation (23.92\%). Similarly, Handiyani et al. (2019) studied hand hygiene among nurses in Indonesia and reported higher compliance in the questionnaire (51.2\%) compared to direct observation (20.4\%) (23). Several other studies have indicated over-reporting when the questionnaire technique is used. According to Nematian et al. (2017) in Iran, overall compliance by observation was low (39.6\%), whereas selfreported compliance was more than 75\% (24). Additionally, Oliveira et al. (2017) from Brazil compared compliance in ICU obtained through direct observation and self-reports among HCWs. They found observed compliance of $19.0 \%$ of 1,935 hand hygiene opportunities, whereas self-reported compliance was $87.9 \%$ (25). Therefore, over-reporting of hand hygiene compliance remains a major concern globally that should be addressed as a key step towards improving hand hygiene compliance.

Similar to other studies, HCWs complied with hand hygiene better after than before patient contact (2628). Researches indicate HCWs tend to be more concerned about protecting themselves than patients from possible microbial infection $(29,30)$. Nevertheless, HCWs often poorly practice hand hygiene after touching patient surroundings due to the lack of awareness with the guidelines that address the indications $(5,31)$. This suggests that some HCWs believe performing hand hygiene after touching patient surroundings is less critical.

HCWs considered social influences such as social support by coworkers, administrators, leaders, role models, patients, and organization culture as a barrier to compliance. Similarly, studies in Vietnam and England found that lack of patient involvement was a possible factor contributing to poor compliance among HCWs $(32,33)$. However, Smith et al. (2019) in Canada and Sendall et al. (2019) in Australia inform that the presence of role models significantly led to the improvement of compliance among HCWs $(12,34)$. We, therefore, recommend promoting role modeling, leadership, and patient involvement in hand hygiene in healthcare settings.

More than $85 \%$ of the HCWs claimed to have a high level of hand hygiene knowledge, and this could be the primary facilitator of hand hygiene. Similarly, McAteer et al. (2014) from England qualitatively used 
psychological theory to investigate barriers and facilitators for the implementation of hand hygiene. They reported that HCWs had high levels of hand hygiene knowledge, therefore, facilitating compliance (33). Additionally, Le et al. (2019) in Vietnam reported comparable results that HCWs had good knowledge of hand hygiene guidelines (32). However, our finding was inconsistent with Shehu et al. (2019) in Nigeria, who reported that only $16 \%$ of HCWs had good knowledge of hand hygiene, due to inadequate training (35). The majority of the HCWs perceived memory, attention, and decision processes hindered hand hygiene compliance. This domain involves the ability to think, forget, and presence of reminders such as posters and signs for hand hygiene, how much attention paid for hand hygiene, and decisions on whether to comply. Additionally, Pedersen et al. (2017) in the USA found that forgetfulness was the primary barrier to compliance in endoscopy procedure rooms (36). In contrast to this, White et al. (2015) and Seo et al. (2019) reported that verbal and visual reminders facilitate compliance $(37,38)$. Nevertheless, a systematic review across different countries noted that the presence of reminders might slightly improve compliance (39).

A significant proportion of $\mathrm{HCWs}$ felt there were inadequate hand hygiene resources such as handrubs and water supply, therefore considered this domain a barrier to compliance. Our findings were inconsistent with results by White et al. (2015) from Australia in their qualitative study through focus group discussions that found the availability of sinks and detergents was the leading facilitator to compliance (37). Nevertheless, our results support a review study to identify barriers to compliance that reported heavy workload and inadequate hand hygiene resources as the common barriers in developing countries (8). A study in Nigeria (2017) found that an insufficient supply of water and handrubs was the major contributing factor to poor compliance (40). These findings support the report by the WHO (2019) on the global status of water, sanitation, and hygiene (WASH) services in healthcare facilities that there is a need to recognize its importance in preventing infections and saving lives (41). Our findings advocate for the need to improve the supply of the facilities as the top priority to facilitate compliance.

Still, the majority of the HCWs had inferior beliefs about hand hygiene outcomes to self, coworkers, family, patients, or community such as protection from spreading diseases, and skin allergic reactions to handrubs. A similar study by Le et al. (2019) in Vietnam reported allergic reactions to handrubs as a barrier to compliance (32). Our findings were however in contrary to a study by Boscart et al. (2012) from Canada who explored nurses' perceived barriers and enablers to compliance and found that belief about consequences was the major hand hygiene facilitator (42). Additionally, dealing with patients, especially those with high infection risks, have been suggested to encourage compliance in care settings (37).

Social/professional role and identity were reported by the majority of the HCWs to facilitate hand hygiene compliance. This domain was also reported by Smith et al. (2019) from Canada and McAteer et al. (2014) from England as a facilitator to hand hygiene $(33,43)$. We, therefore, believe that HCWs in low resource settings similar to those in developed countries may have a substantial social/professional role and identity, which encourages compliance. 
We did not find other HCWs characteristics such as gender, age, profession, and education to be significantly associated with hand hygiene compliance. Similarly, Bedoya et. al (2017) in Kenya reported a weak association between compliance and HCWs characteristics (14). The findings may reveal that in settings with inadequate facilities, insufficient hand hygiene training, poor social influences, the demographics of HCWs may not be significant factors of compliance because even with good knowledge and attitude, the setting may not be supportive for everyone.

This study had some limitations. The samples involved in the observation and questionnaire were regarded as independent since pairing was not possible, therefore comparing the two sets of compliances may not be accurate. Moreover, some data emanated from self-reported questionnaires attracting possible reporting bias. Still, the Hawthorne effect could have affected the findings, given that we made the observations overtly, some HCWs may have performed hand hygiene better having known they were being observed. This study was done in one department which may limit the generalizability of its findings to other units. Despite these limitations, our study presents hand hygiene behaviors across different professionals in a multifaceted way with a standardized observation approach and behavior change questionnaire survey to allow a clear understanding of the hand hygiene culture.

\section{Conclusions}

Depending only on the HCWs' self-reports on hand hygiene is not reliable and may negatively influence hand hygiene intervention. The barriers such as inadequate social influences, memory, attention, and decision processes, beliefs about consequences, environmental context, and resources should be considered when designing intervention strategies. Even though the majority of the HCWs claimed they were knowledgeable on hand hygiene, regular training is necessary to enhance hand hygiene compliance.

\section{Abbreviations}

HCAls: Healthcare-associated Infections; HCWs: Healthcare Workers; Theoretical Domains Framework; PCA: Patient Care Assistant; ICU: Intensive Care Unit; WHO: World Health Organization; IRB: Institutional Review Board; WASH: Water, Sanitation, and Hygiene.

\section{Declarations}

\section{Ethics approval and consent to participate}

We obtained the authorization of this study from the Institutional Review Board (IRB) at Central South University (IRB approval number: 12019010). Furthermore, hospital leaders gave written support before starting project activities. The heads of the departments notified their members of the study. Involved HCWs were informed of the aim and background of the research and what the researcher required from them. The data collection was anonymous and confidential, and all participants provided oral consent.

\section{Consent for publication}


Not applicable.

\section{Availability of data and materials}

The datasets used and/or analyzed during this study are available from the corresponding author on reasonable request.

\section{Competing Interests}

The authors declare that they have no competing interests.

\section{Funding}

No funding received

\section{Author's contributions}

KK: Designed the study, collected, and analyzed data. AC: Analyzed data. MK: drafted of the manuscript. WH: Designed the study and critically reviewed the manuscript's draft: All authors read and approved the final manuscript.

\section{Acknowledgments}

The authors thank all the participants in the research, heads of departments, and administration at Kapsabet County Referral hospital, Kenya, for their vital contributions to this work.

\section{References}

1. Chatfield SL, DeBois K, Nolan R, Crawford H, Hallam JS. Hand hygiene among healthcare workers: A qualitative meta summary using the GRADE-CERQual process. Journal of infection prevention. 2017;18(3):104-20.

2. WHO Guidelines Approved by the Guidelines Review Committee. WHO Guidelines on Hand Hygiene in Health Care: First Global Patient Safety Challenge Clean Care Is Safer Care. Geneva: World Health Organization; 2009

3. Office NA. Reducing healthcare-associated infections in hospitals in England: The Stationery Office; 2009.

4. Vos MC, Memish ZA. The Healthcare Worker as a Source of Transmission.

5. Boyce J, Chartier Y, Chraiti M, Cookson B, Damani N, Dharan SJGWHO. WHO guidelines on hand hygiene in health care. 2009.

6. Korniewicz DM. Infection Control for Advanced Practice Professionals: DEStech Publications, Inc; 2013. 
7. Weston D. Fundamentals of infection prevention and control: theory and practice: John Wiley \& Sons; 2013.

8. Ataiyero Y, Dyson J, Graham M. Barriers to hand hygiene practices among health care workers in subSaharan African countries: A narrative review. Am J Infect Control. 2019;47(5):565-73.

9. Kelcikova S, Mazuchova L, Bielena L, Filova L. Flawed self-assessment in hand hygiene: A major contributor to infections in clinical practice? J Clin Nurs. 2019;28(11-12):2265-75.

10. Chavali S, Menon V, Shukla U. Hand hygiene compliance among healthcare workers in an accredited tertiary care hospital. Indian J Crit Care Med. 2014;18(10):689-93.

11. Pittet D, Allegranzi B, Boyce J. The World Health Organization Guidelines on Hand Hygiene in Health Care and their consensus recommendations. Infect Control Hosp Epidemiol. 2009;30(7):611-22.

12. Smith J, Corace K, MacDonald T, Fabrigar L, Saedi A, Chaplin A, et al. Application of the Theoretical Domains Framework to identify factors that influence hand hygiene compliance in long-term care. 2019;101(4):393-8.

13. Fuller C, Besser S, Savage J, McAteer J, Stone S, Michie S. Application of a theoretical framework for behavior change to hospital workers' real-time explanations for non-compliance with hand hygiene guidelines. American journal of infection control. 2014;42(2):106-10.

14. Bedoya G, Dolinger A, Rogo K, Mwaura N, Wafula F, Coarasa J, et al. Observations of infection prevention and control practices in primary health care, Kenya. 2017;95(7):503.

15. Ngugi SK, Murila FV, Musoke RNJJoIP. Hand hygiene practices among healthcare workers in a newborn unit of a tertiary referral hospital in Kenya. 2019;20(3):132-8.

16. Ndegwa L, Hatfield KM, Sinkowitz-Cochran R, D'lorio E, Gupta N, Kimotho J, et al. Evaluation of a program to improve hand hygiene in Kenyan hospitals through production and promotion of alcoholbased Handrub-2012-2014. 2019;8(1):1-6.

17. World Health O, Safety WHOP. Hand hygiene technical reference manual: to be used by health-care workers, trainers, and observers of hand hygiene practices. Geneva: World Health Organization; 2009.

18. Organization WHOJWH. Training Film: A tool to help convey the concept of the" 5 moments for hand hygiene" to health-care workers. 2016.

19. Kohli E, Ptak J, Smith R, Taylor E, Talbot EA, Kirkland KB. Variability in the Hawthorne effect with regard to hand hygiene performance in high- and low-performing inpatient care units. Infect Control Hosp Epidemiol. 2009;30(3):222-5.

20. Chen LF, Vander Weg MW, Hofmann DA, Reisinger HS. The Hawthorne Effect in Infection Prevention and Epidemiology. Infect Control Hosp Epidemiol. 2015;36(12):1444-50.

21. Ahmed N, Taneepanichskul SJJoHR. Knowledge, Attitude and Practice of Dengue Fever Prevention

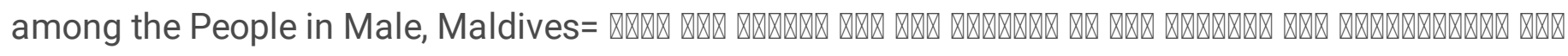

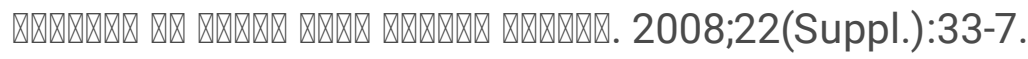

22. Azfar M, Omarulharis S, Azfar H, Maryam A, Hafizah S, Adibah B, et al. Knowledge, attitude and practice of dengue prevention among sub urban community in Sepang, Selangor. 2017;4(2):73-83. 
23. Handiyani $H$, Ikegawa $M$, Hariyati RTS, Ito $M$, Amirulloh $F$. The determinant factor of nurse's hand hygiene adherence in Indonesia. Enferm Clin. 2019;29 Suppl 2:257-61.

24. Nematian SSS, Palenik CJ, Mirmasoudi SK, Hatam N, Askarian MJAjoic. Comparing knowledge and self-reported hand hygiene practices with direct observation among Iranian hospital nurses. 2017;45(6):e65-e7.

25. Oliveira AC, de Paula AO, Gama CSJEG. Monitoring hand hygiene: direct observation versus selfreport rates. 2017;16(4):344-53.

26. Allegranzi B, Gayet-Ageron A, Damani N, Bengaly L, McLaws ML, Moro ML, et al. Global implementation of WHO's multimodal strategy for improvement of hand hygiene: a quasiexperimental study. Lancet Infect Dis. 2013;13(10):843-51.

27. Haac B, Rock C, Harris AD, Pineles L, Stein D, Scalea T, et al. Hand hygiene compliance in the setting of trauma resuscitation. 2017;48(1):165-70.

28. Muller MP, Carter E, Siddiqui N, Larson EJAEM. Hand hygiene compliance in an emergency department: the effect of crowding. 2015;22(10):1218-21.

29. Lee A, Chalfine A, Daikos GL, Garilli S, Jovanovic B, Lemmen S, et al. Hand hygiene practices and adherence determinants in surgical wards across Europe and Israel: a multicenter observational study. 2011;39(6):517-20.

30. Cheng VC, Tai JW, Ho SK, Chan JF, Hung KN, Ho PL, et al. Introduction of an electronic monitoring system for monitoring compliance with Moments 1 and 4 of the WHO" My 5 Moments for Hand Hygiene" methodology. 2011;11(1):151.

31. Aboumatar $H$, Ristaino $P, D$ avis RO, Thompson CB, Maragakis L, Cosgrove S, et al. Infection prevention promotion program based on the PRECEDE model: improving hand hygiene behaviors among healthcare personnel. 2012;33(2):144-51.

32. Le CD, Lehman EB, Nguyen TH, Craig TJJljoer, health $\mathrm{p}$. Hand hygiene compliance study at a large central hospital in Vietnam. 2019;16(4):607.

33. McAteer J, Stone S, Fuller C, Michie SJAjoic. Using psychological theory to understand the challenges facing staff delivering a ward-led intervention to increase hand hygiene behavior: a qualitative study. 2014;42(5):495-9.

34. Sendall MC, McCosker LK, Halton KJljoer, health p. Cleaning staff's attitudes about hand hygiene in a metropolitan hospital in Australia: A qualitative study. 2019;16(6):1067.

35. Shehu N, Onyedibe K, Igbanugo J, Okolo M, Gomerep S, Isa S, et al. Hand hygiene knowledge, training and practice: A cross-sectional study in a tertiary health institution, North-central Nigeria. 2019;22(7):1008.

36. Pedersen L, Elgin K, Peace B, Masroor N, Doll M, Sanogo K, et al. Barriers, perceptions, and adherence: Hand hygiene in the operating room and endoscopy suite. American journal of infection control. 2017;45(6):695-7.

37. White KM, Jimmieson NL, Obst PL, Graves N, Barnett A, Cockshaw W, et al. Using a theory of planned behaviour framework to explore hand hygiene beliefs at the ' 5 critical moments' among Australian 
hospital-based nurses. 2015;15(1):59.

38. Seo H-J, Sohng K-Y, Chang SO, Chaung SK, Won J, Choi M-JJJoHI. Interventions to improve hand hygiene compliance in emergency departments: a systematic review. 2019.

39. Gould DJ, Moralejo D, Drey N, Chudleigh JH, Taljaard MJCdosr. Interventions to improve hand hygiene compliance in patient care. 2017(9).

40. Ango UM, Awosan KJ, Adamu H, Salawu S, Sani MM, Ibrahim AJIJTDH. Knowledge, attitude and practice of hand hygiene among healthcare providers in semi-urban communities of Sokoto State, Nigeria. 2017;26:1-9.

41. Organization WH. Water, sanitation and hygiene in health care facilities: practical steps to achieve universal access to quality care. 2019.

42. Boscart VM, Fernie GR, Lee JH, Jaglal SB. Using psychological theory to inform methods to optimize the implementation of a hand hygiene intervention. Implement Sci. 2012;7:77.

43. Smith JD, Corace KM, MacDonald TK, Fabrigar LR, Saedi A, Chaplin A, et al. Application of the Theoretical Domains Framework to identify factors that influence hand hygiene compliance in longterm care. The Journal of hospital infection. 2019;101(4):393-8. 